\title{
FATORES DE RISCO PARA HIPERTENSÃO ARTERIAL SISTÊMICA VERSUS ESTILO DE VIDA DOCENTE
}

RISK FACTORS FOR ARTERIAL SYSTEMIC HYPERTENSION VERSUS PROFESSORS' LIFE STYLE

FACTORES DE RIESGO PARA HIPERTENSIÓN ARTERIAL SISTÉMICA VERSUS ESTILO DE VIDA DOCENTE

\author{
Jorge Luiz Lima da Silva ${ }^{1}$ \\ Solange Lourdes de Souza ${ }^{2}$
}

RESUMO: A hipertensão arterial sistêmica (HAS) representa grave problema de saúde. Alguns fatores de risco para a doença são mais comuns em centros urbanos das metrópoles. Como há uma relação direta do estilo de vida com os fatores de risco, perscrutou-se o estilo de vida dos profissionais da docência de uma universidade pública, a fim de detectar os fatores de risco para HAS.O estudo tem natureza descritiva, ancorado na pesquisa qualitativa. Objetivouse identificar fatores de risco no estilo de vida dos docentes, e conhecer e classificar o nível pressórico do grupo. Os resultados apontaram fatores de risco para HAS em grande parte dos atores sociais e pressão sanguínea acima dos índices recomendados pelo Ministério da Saúde e organizações de estudo do coração e de hipertensão. Conclui-se que para o corpo docente é necessária mudança no estilo de vida, o que é surpreendente, pois o grupo ensina como cuidar de pessoas.

PALAVRAS-CHAVES: Hipertensão; Estilo de Vida; Fatores de Risco.

ABSTRACT: The systemic arterial hypertension (SAH) represents serious problem of health. Some risk factors for illness are more common in great urban centers. Because there is a direct interrelation between life style with risk factors to $\mathrm{SAH}$, it was inquired about life style of public university professors to detect risk factors to SAH. This study has descriptive nature, on qualitative research. The objective was to identify the factors of risk for SAH in the style of life of the professors, and to know and to classify the pressure level of the group. The results points to risk factors to SAH for most professors and blood pressure higher than Health Ministry and heart and blood pressure specialized institutions recommends. Concluding, that's necessary for professors to change their lifestyle, specially because they teach how to take care of other human being.

\section{KEYWORDS : Hypertension; Life Style of Life, Risk Factors.}

RESUMEN: La hipertensión arterial sistemática (HAS) representa grave problema de salud. Algunos factores de riesgo para la enfermedad son más comunes en centros urbanos de las metrópolis. Como hay una relación directa del estilo de vida con los factores de riesgo, se investigó el estilo de vida de los profesionales de la docencia de una universidad pública, la fin de detectar los factores de riesgo para HAS. El estudio tiene naturaleza descriptiva, anclado en la búsqueda cualitativa. Objetivo identificarse factores de riesgo en el estilo de vida de los docentes, y conocer y clasificar el nivel pressorico del grupo. Los resultados apuntaron factores de riesgo para HAS en gran medida de los actores sociales y presión sanguínea arriba de los índices recomendados por el Ministerio de la Salud y organizaciones de estudio del corazón y de hipertensión. Se concluye que para el cuerpo docente es necesario cambio en el estilo de vida, el que es sorprendente, pues el grupo enseña como cuidar de personas.

TERMINOS CLAVES: Hipertensión; Estilo de Vida; Factores de Riesgo.

\footnotetext{
${ }^{1}$ Professor Substituto do Departamento de Fundamentos de Enfermagem e Administração da Escola de Enfermagem Aurora de Afonso Costa da Universidade Federal Fluminense.Pós-graduando em formação pedagógica em educação profissional na área da saúde pela Escola Nacional de Saúde Pública/ Fundação Oswaldo Cruz (ENSP/FIOCRUZ). E-mail: jorgeluizlima@click21.com.br

2 Professora Adjunta IV, do Departamento de Enfermagem Materno-Infantil e Psiquiátrica da Escola de Enfermagem Aurora de Afonso Costa da Universidade Federal Fluminense. Mestre em enfermagem. E-mail: solgueiviju@hotmail.com
} 


\section{INTRODUÇÃO}

O presente artigo foi constituído a partir de parte de monografia de conclusão de curso a qual seguiu os parâmetros da instituição acadêmica para a obtenção do título de enfermeiro e licenciatura em enfermagem (SILVA, 2004).

A hipertensão arterial sistêmica (HAS) representa grave problema de saúde no país, não só pela elevada prevalência - cerca de $20 \%$ da população adulta como também pela acentuada parcela de hipertensos não diagnosticada, ou não tratada de forma adequada, ou ainda pelo alto índice de abandono ao tratamento.

Hipertensão arterial é uma síndrome clínica caracterizada pela elevação da pressão arterial a níveis iguais ou superiores a $140 \mathrm{~mm} \mathrm{Hg}$ de pressão sistólica e/ ou $90 \mathrm{~mm} \mathrm{Hg}$ de diastólica — em pelo menos duas aferições subseqüentes - obtidas em dias diferentes, ou em condições de repouso e ambiente tranqüilo. Quase sempre, acompanham esses achados de forma progressiva, lesões nos vasos sanguíneos com conseqüentes alterações de órgãos alvos como cérebro, coração, rins e retina. Geralmente, é uma doença silenciosa: não dói, não provoca sintomas, entretanto, pode matar. Quando ocorrem sintomas, já decorrem de complicações.

A classificação utilizada, mais recente, é preconizada pela Sociedade Brasileira de Cardiologia baseada em parâmetros norte americanos (CAMPOS JR. et al, 2001). Houve uma simplificação das faixas pressóricas e a categorização de uma situação dita "préhipertensão", onde as modificações do estilo de vida devem ser mais que incentivadas, tendo em vista a grande possibilidade de evolução futura para o estado de hipertensão arterial com o avançar da idade. Nesta classificação atual, a pressão ideal é aquela menor que 120 sistólica e 80 diastólica. O Ministério da Saúde (MS) considera este valor ideal, onde há menos riscos para o aparelho cardiovascular.(BRASIL, 2002, p.13)

\section{Questões norteadoras e objetivos}

$\mathrm{Na}$ abordagem do estudo da pressão arterial sistêmica; deve-se levar em conta a história familiar e os hábitos de vida, nesta perspectiva, o estudo norteou-se pelas seguintes questões: Quais seriam os fatores de risco para a doença inseridos no estilo de vida de docentes? Qual é a média do índice pressórico entre os docentes da Escola de Enfermagem?

O estudo teve como objetivos identificar os fatores de risco para HAS no estilo de vida dos docentes e conhecer e classificar o nível pressórico do grupo de docentes pesquisados. Desta forma, contemplou-se o objeto de estudo que é o estilo de vida docente e os fatores de risco para HAS.

A relevância do estudo está em sua permanência conteudística, e soma-se a este fator o caráter detectivo e preventivo, o que enseja a ser uma oportunidade de reflexão para a população pesquisada.

\section{METODOLOGIA}

O estudo foi embasado nas normas de uma pesquisa descritiva, com abordagem qualitativa. Com base nas palavras de RUDIO (2001), descrever é narrar o que acontece e, desta forma, a pesquisa descritiva está interessada em descobrir o que acontece; conhecer o fenômeno, procurando interpretá-lo, e descrevê-lo.

De acordo com TOBAR \& YALOUR (2002), uma pesquisa qualitativa deve envolver múltiplas fontes de dados, empregar a observação de primeira mão, interessar-se pelo cotidiano, situar-se num contexto de descobrimento, importar-se mais com os significados do que com a freqüência dos fatos e deve buscar o específico e o local para encontrar padrões, não estando atado ao modelo teórico.

Os dados, para serem interpretados e observados de forma plena em características específicas do estilo de vida, tiveram que ser quantificados inicialmente para que depois se processasse a análise de conteúdo que, "em sua história mais recente, isto é, enquanto técnica de tratamento dos dados considerada cientificamente, é caudatária das metodologias quantitativas, buscando sua lógica na interpretação cifrada do material de caráter qualitativo." (MINAYO, 2000, p.202) Procedeu-se à análise com dados numéricos; mas que, no geral, formaram características mais amplas que são os hábitos e costumes, quando somados indicaram um padrão e estilos, para que em seguida fossem agrupados e caracterizados.

Para a coleta de dados foi utilizado o questionário que constou de três quadros divididos por assuntos. O quadro I indagou sobre o histórico familiar e se era portador de HAS. Quadro II abordou os fatores de risco. Quadro III é referente às medidas do corpo e hábitos alimentares. Ao final, há uma lacuna para inserir os valores pressóricos mensurados. Este último item foi coletado duas vezes em semanas diferentes, seguindo as normas de aferição da pressão arterial (PA) segundo o III Consenso Brasileiro de Hipertensão Arterial (CBHA) (BRASIL, 2001).

\section{Os sujeitos da pesquisa}

Os sujeitos da pesquisa foram 23 professores, constituindo a amostra randômica, pois se objetivou conhecer o estilo de vida do grupo, destacando os fatores de risco para hipertensão arterial. Estabeleceram-se critérios de que os docentes afastados para curso de mestrado, doutorado ou aposentados não poderiam participar da amostragem. Elegeu-se um grupo heterogêneo porque, desta forma, conseguiu-se visualizar as diversidades e as 
similaridades existentes entre indivíduos que estão inseridos em uma rotina de vida em seu trabalho.

Cada participante preencheu o consentimento livre e esclarecido, pois em se tratando de pesquisa envolvendo seres humanos o Conselho Nacional de Saúde (Resolução CNS 196/96, de 16 de outubro de 1996) exige que o sujeito integrante do grupo a ser pesquisado conheça os objetivos do estudo e dê consentimento às regras do mesmo (BRASIL, 1996). O projeto de pesquisa foi aprovado em primeira instância pelo comitê de ética em pesquisa do Hospital Universitário, sendo este, reconhecido pelo CONEP.

\section{O tratamento dos dados}

Os dados coletados foram analisados e agrupados em duas categorias. Após análise à luz do referencial teórico abordado pelo estudo (vide bibliografia), buscou-se relacionar os hábitos diários de docentes com os fatores de risco predisponentes para HAS. "A palavra categoria, em geral, se refere a conceito que abrange elementos ou aspectos com características comuns ou que se relacionam entre si. [...] As categorias são empregadas para se estabelecer classificações." (MINAYO, 2001, p.70)

As duas categorias surgidas reuniram informações sobre os fatores de risco e nível pressórico dos docentes.

$\mathrm{Na}$ primeira categoria encontramos aspectos sobre os fatores de risco não modificáveis: a idade, sexo masculino e hereditariedade. São características que constituem o indivíduo sendo peculiar e intrínseco, não sendo modificáveis, contudo mensuráveis e passíveis de análise interpretativa. A segunda categoria traz os fatores de risco modificáveis. Nesta última, surgem os hábitos sociais e uso de anticoncepcionais, padrões alimentares e aspectos físicos favoráveis à gênese da HAS. Na terceira categoria, encontram-se os valores pressóricos mensurados, sua classificação e correlação com o estilo de vida docente encontrado.

\section{RESULTADOS E DISCUSSÃO}

Conforme definição abordada ROUQUAYROL \& FILHO (1999, p.288), estilo de vida são: "hábitos e comportamentos auto-determinados, adquiridos social ou culturalmente, de modo individual ou em grupo". Compreende-se aqui que, no decorrer da análise dos hábitos foram colocados em evidência os fatores de risco, pois estes são integrantes do estilo de vida e identificáveis neste mesmo estilo.

\section{Fatores de risco não modificáveis}

- Idade:

Quanto à idade, todos os indivíduos encontram-se na faixa dos 40 anos ou mais (100\%). Percebe-se, através da análise de conteúdos de literatura científica, que o aumento da pressão com o avançar da idade tem sido observado, embora segundo III CBHA (BRASIL, 2001) indique que esse aumento não represente um comportamento fisiológico normal. Preveni-lo constitui o meio mais eficiente de combater a hipertensão arterial, evitando as dificuldades e o elevado custo social de seu tratamento e de suas complicações. Para LESSA (1998), a presença de HAS em idosos merece maior atenção devido à vulnerabilidade frente às complicações cardiovasculares determinadas não só pela hipertensão, como também por outros fatores de risco que se acumulam com o passar do tempo.

\section{- Hereditariedade:}

O caráter hereditário aparece em $74 \%$ dos sujeitos. Para BARRETO-FILHO \& KRIEGER (2003), dos fatores envolvidos na fisiopatogênese da hipertensão arterial, um terço deles pode ser atribuído a fatores genéticos. Citam como exemplo o sistema regulador da pressão arterial e sensibilidade ao sal.

Os autores deixam claro que a hipertensão arterial pode ser entendida como uma síndrome multifatorial, de patogênese pouco elucidada, na qual interações complexas entre fatores genéticos e ambientais causam elevação sustentada da pressão. Em aproximadamente $90 \%$ a $95 \%$ dos casos não existe etiologia conhecida ou cura, sendo o controle da pressão arterial obtido por mudanças do estilo de vida e tratamento farmacológico.

\section{- Sexo:}

Dos sujeitos da pesquisa, somente um era do sexo masculino o que corresponde a $4,35 \%$. Como houve uma predominância do sexo feminino, fez-se por relevante abordar a relação entre climatério e HAS onde IRIGOYEN et al (2003) afirmam que estudos demonstraram que a pressão arterial é mais elevada em homens que em mulheres até a faixa etária de 60 anos. Sugere-se que os hormônios ovarianos são responsáveis pela pressão mais baixa nas mulheres (durante o climatério) e com a chegada da menopausa a prevalência da pressão alta entre homens e mulheres tende a se aproximar.

\section{Os fatores de risco modificáveis}

\section{- Hábitos sociais e uso de anticoncepcionais:}

Anticoncepcionais: $34,7 \%$ dos sujeitos da pesquisa fizeram ou fazem uso de contraceptivo hormonal. O III CBHA (BRASIL, 2001) afirma que a ingestão de anticoncepcionais orais deve ser considerada como possível causa de hipertensão arterial. Embora não haja contra-indicação formal, o "uso de anticoncepcionais orais deve ser evitado em mulheres com mais de 35 anos de idade e em obesas, 
pelo maior risco de hipertensão arterial. Em mulheres com mais de 35 anos e fumantes irredutíveis, o anticoncepcional oral está formalmente contra-indicado" (BRASIL, 2001, p. 28)

Tabagismo: $82,3 \%$ não fumam. $28,7 \%$ já fumaram ou fumam. É evidente que os efeitos do tabagismo são maléficos em curto ou longo prazo para saúde. O fator vasoconstricção é o mais relevante, além de acelerar o processo de arteriosclerose. Geralmente, o fumante busca o famoso "cafezinho", nas situações estressantes e também condicionam ao consumo de cafeína. OPARIL (1997, p.291) afirma que "a cafeína e a nicotina elevam agudamente a PA". Segundo o III CBHA (BRASIL, 2001), o tabagismo colabora para o efeito adverso da terapêutica de redução dos lípides séricos e induz resistência ao efeito de drogas anti-hipertensivas.

Bebida alcoólica: $65 \%$ declararam-se não consumidores de bebidas alcoólicas, enquanto $35 \%$ consomem socialmente. "O consumo de álcool eleva a PA tanto agudo quanto cronicamente" (OPARIL, 1997, p. 290) "Padrões de consumo e comportamento são os principais fatores de risco para HA. Destacam-se o consumo excessivo de calorias e de bebidas alcoólicas, a inatividade física, a baixa ingestão de potássio e o elevado consumo de sódio".(LESSA, 1998, p.84).

\section{- Padrões alimentares e aspectos físicos}

Sedentarismo e obesidade: Os dados mostram que $39,1 \%$ não praticam atividade física regularmente; contra os $71,9 \%$ que praticam alguma atividade física com regularidade. Entretanto, é necessário avaliar a freqüência e intensidade desses exercícios referidos pelos docentes já que 56,52 \% estão acima do peso, considerando IMC ideal até 25, segundo BRASIL (2002, p.22). Destes, $38 \%$ já são considerados obesos moderados classe 1(IMC entre 30 - 34,9), segundo classificação da OMS. O risco de co-morbidade é moderado o que inclui HAS.

Hábitos alimentares: Quanto ao número de refeições, grande parte do grupo (39,4 \%) informou que faz 4 refeições ao dia e 4,3\% fazem uma refeição ao dia. O mais importante sobre a alimentação não é o número de vezes, mas sim a qualidade do que é ingerido. Sal, refrigerantes, charque e massas estão no cardápio dos docentes.

Quando não é possível uma alimentação adequada, é substituída por lanches que, do ponto de vista nutricional, deixam a desejar, além de serem uma boa fonte de calorias e gorduras saturadas. Assim como os salgadinhos de pacote que além do sal, em sua maioria, contém massa na composição. Salgadinhos de pacote são consumidos esporadicamente por $52 \%$ e recusados por $39 \%$ e está no consumo freqüente de $9 \%$.

$\mathrm{O}$ consumo freqüente de enlatados apareceu em $65,21 \%$ como raramente, e $13,3 \%$ que consomem freqüentemente. Importante quantificar devido à grande quantidade de condimentos - dentre eles o sal - e calorias encontradas em enlatados e conservas. Nos grandes centros urbanos, é comum o consumo de enlatados e conservas devido à praticidade para o consumo. Por sua vez, o consumo freqüente de conservas apareceu em $78,26 \%$ onde $6,65 \%$ consomem freqüentemente.

O consumo de massas é de $100 \%$ aparecendo em $69,56 \%$ com freqüência e esporadicamente para $30,44 \%$

Leite e seus derivados são relatados por $100 \%$ dos sujeitos da pesquisa como consumidos freqüentemente. Tais alimentos são ricos em cálcio que por um lado é bom, pois "estudos epidemiológicos sugeriram uma relação inversa entre a ingestão dietética de cálcio e a HAS: os hipertensos consomem menos cálcio do que as pessoas normotensas" (OPARIL, 1997, p.291). Deve-se consumir queijo branco (rico em cálcio) e leite desnatado que possui menos gorduras saturadas.

$53,52 \%$ admitem consumir freqüentemente doces, enquanto $26,08 \%$ consomem doces raramente. $20,40 \%$ não consumem doces.

A carne salgada (carne de sol, ou carne seca) é consumida esporadicamente por $69 \%$ dos sujeitos, enquanto $13 \%$ consomem com freqüência e $17 \%$ não consomem.

As carnes vermelha e branca são consumidas freqüentemente por $100 \%$ dos sujeitos sendo que $65 \%$ consomem freqüentemente os dois tipos de carne. Uma dieta básica para o controle/ profilaxia da HAS inclui $90 \mathrm{~g}$ de carne branca peixe ou ave como afirmam NETTINA et al (2003).

A carne vermelha contém mais gorduras saturadas. O estudo de LOPES (2003, p.68) demonstra uma dieta rica em ácidos graxos não esterificados que atuam no sistema nervoso central, responsável pela liberação de substâncias vasoconstritoras, o que pode resultar no aumento da pressão pelo menos agudamente.

Os especialistas recomendam 9 gramas de sal por dia que pode ser conseguido naturalmente durante uma refeição se não for controlado o uso de cloreto de sódio. $22 \%$ utilizam o saleiro freqüentemente durante as refeições; $18 \%$ utilizam raramente o saleiro; $60 \%$ não utilizam o saleiro.

Muitos docentes moram distante de casa, o que faz com que 39,14\% alimentem-se na rua. A quantidade de sal utilizada no preparo dos alimentos em restaurantes não é informada, ao se utilizar o saleiro, acrescenta-se mais sal a alimentos que naturalmente já contém sódio em quantidade ideal.

$60,86 \%$ admitem beber refrigerantes durante as refeições destes $71,4 \%$ dizem que é com pouca freqüência, enquanto $28,6 \%$ admitem consumir diariamente. Os refrigerantes contêm, em grande parte de sua composição, açúcar fonte de calorias concentradas $(200 \mathrm{ml}=90$ calorias $)$. (ibid)

O mundo contemporâneo com dietas industrializadas, ricas em sódio, carboidratos e colesterol 
são fatores de risco não só para a hipertensão arterial bem como obesidade, dislipidemia e diabetes. Estes fatores agravados pelo sedentarismo favorecem a elevação de riscos para as complicações tardias e imediatas da doença. O aspecto físico somado aos hábitos alimentares inadequados indica uma dieta hipersódica e hipercalórica que contribuem para elevação da pressão.

\section{- Índices pressóricos encontrados}

Para melhor compreensão, os índices pressóricos encontrados foram classificados de acordo com os novos parâmetros para avaliação da pressão arterial, onde se considera o índice entre 120 - $139 \mathrm{~mm} \mathrm{Hg}$ para PA sistólica e entre $80-89 \mathrm{~mm} \mathrm{Hg}$ para PA diastólica como pré-hipertensão; ressalta-se assim, serem necessárias mudanças no estilo de vida a fim de se evitar um possível desenvolvimento/ surgimento da afecção sindrômica

Foi constatado que $24 \%$ dos sujeitos da amostra eram portadores de HAS (declarados) e $86 \%$ afirmavam não serem portadores. Do grupo que afirmava não ter índices pressóricos elevados, $10 \%$ possuíam valores acima do teto ideal preconizado pelo MS.

O índice de pré-hipertensos (PA entre $120 \times 80$ e $139 \times 89 \mathrm{~mm} \mathrm{Hg}$ ) foi de $47,8 \%$ o que alerta para o controle dos hábitos que constituem fatores de risco para o desenvolvimento da doença que mata silenciosamente. NETTINA et al (2003, p.415) considera a PA até $120 \times 80 \mathrm{~mm} \mathrm{Hg}$ ótima mencionado que os riscos cardiovasculares estão acima desse valor.

Segundo LESSA (1998), a hipertensão arterial como entidade isolada é encontrada como a mais freqüente causa de morbidade do adulto em todo o mundo industrializado, na sua maioria em países em desenvolvimento, sobretudo nos grandes centros urbanos.

\section{- Classificando as cifras}

Considerando PA até $120 \times 80 \mathrm{~mm} \mathrm{Hg}$ (índice preconizado pelo MS atualmente como PA ideal) e pelo III CBHA temos $52 \%$ de indivíduos acima do ideal, o que somados aos hábitos de vida e fatores de vida podem desencadear o surgimento da síndrome em índices pressóricos mais elevados (BRASIL, 2001). Deste grupo, somente $16 \%$ apresentaram as duas pressões (sistólica e diastólica) até patamar ideal. Demais indivíduos apresentaram valores até os ideais isolados. Ora PA sistólica $\geq 120 \mathrm{~mm} \mathrm{Hg}$, ora diastólica $\geq$ $80 \mathrm{~mm} \mathrm{Hg}$ (considerando as duas mensurações-padrão e a média entre elas).

Apesar de não compor o estilo de vida, encontrase a influência de fatores hereditários e idade, os quais se destacaram como possíveis fatores contribuintes para HAS, estando à mercê das influências ambientais que possuem importante papel no que diz respeito à expressão genética.

$\mathrm{Na}$ análise dos fatores de risco e grau de classificação da HA, segundo MS, $21,7 \%$ dos docentes estão no grupo de risco médio, onde há indivíduos com um ou dois fatores de risco cardiovascular. "Alguns possuem baixos níveis de pressão arterial e múltiplos fatores de risco, enquanto outros possuem altos níveis de pressão arterial e nenhum, ou poucos fatores de risco. Entre os indivíduos desse grupo, a probabilidade de um evento cardiovascular grave, nos próximos dez anos, situa-se entre 15 e 20\%”.(BRASIL, 2002 p.19)

De acordo com estratificação em grupos seguindo os fatores de risco (BRASIL, 2001), os docentes se encontram no grupo $B$, onde há presença de fatores de risco (não incluindo diabetes Mellitus) e sem lesão de órgãos-alvo. A conduta é modificação no estilo de vida.

Desta forma, constata-se que a PA de 21, $7 \%$ encontra-se em risco maior de complicações nos próximos dez anos, segundo afirmações do MS. De acordo com os novos parâmetros americanos $(\mathrm{NIH}$, 2003) esta porcentagem encontra-se no grupo de préhipertensos e a conduta é semelhante a do MS.

O conteúdo de maior relevância desta da análise está nos aspectos modificáveis, por se inserir neste âmbito o estilo de vida. Todavia, os aspectos não modificáveis serão mencionados devido ao sinergismo entre fatores de risco que são cruciais na manifestação da doença hipertensiva.

O ritmo de vida constante, a uma má-alimentação (fora do horário, corrida, improvisada) e dieta não muito favorável à manutenção da saúde, o consumo de bebida alcoólica, tabagismo e uso de anticoncepcionais também integram o estilo de vida, pois são hábitos ou costumes, mas que se destacaram como fatores de risco para HAS, somando-se ainda as características da dieta que é rica em calorias e sódio.

Apesar de não compor o estilo de vida, encontrase a influência de fatores hereditários e idade, os quais se destacaram como possíveis fatores contribuintes para HAS, estando à mercê das influências ambientais que possuem importante papel no que diz respeito à expressão genética.

Ganha destaque o gênero feminino o qual se encontra no climatério, talvez seja o grupo mais acometido pelo estresse, pois necessita de tempo para diversos afazeres incluindo os domésticos. O estresse ganha espaço e foi analisado em um estudo complementar, pois IRIGOYEN (2003) afirma que é difícil estabelecer um papel mais definido ao estresse na gênese da HAS, devido a este fator estar ligado a outros dentre eles o sedentarismo, dieta e hábitos sociais.

\section{CONCLUSÕES}

Hábitos alimentares prejudiciais, consumo de bebidas alcoólicas, tabagismo, obesidade e consumo de 
anticoncepcionais são os que mais aparecem somados na análise dos dados. Quando confrontados com 47,8\% de docentes com pressão normal limítrofe julga-se que o fato é alarmante e surpreendedor, já que os sujeitos da pesquisa são professores que ensinam a cuidar de pessoas.

Considerações a respeito do sobrepeso devem ser direcionadas à dieta, apontando para a necessidade de reeducação alimentar e não dietas curtas. Considerase a dieta como a primeira conduta, pois funciona como o principal fator relevante dentro dos fatores modificáveis. Após o controle deste, não há uma resolução completa dos fatores modificáveis, até porque o grupo de pessoas aqui relacionado convive no mundo pós-moderno e tecnológico, onde a acomodação é quase que um convite.

Para que o autocuidado tenha sucesso é necessário perceber as próprias necessidades, ou seja, indagar-se sobre que é realmente preciso ter no estilo de vida para manter a saúde. A percepção do indivíduo sobre um problema a ser enfrentado, em seu ritmo natural, é um fator importante que influencia na reação para a busca de melhorias. A partir deste ponto, há possibilidade de harmonizar a saúde com o viver do cotidiano.

Percebe-se que os hábitos sociais encontrados estão diretamente relacionados com os índices pressóricos encontrados, já que são classificados pela literatura pertinente como fatores de risco em potencial para o desenvolvimento de HAS.

$O$ estudo em comento traz, portanto, aos docentes, um alerta de mudança de estilo de vida, para a qualidade da própria vida.

\section{REFERÊNCIAS BIBLIOGRÁFICAS}

BARRETO-FILHO, J. A. S; KRIEGER, J. E. Genética e hipertensão arterial: conhecimento aplicado à prática clínica. Rev. Soc. Bras. Card. Estado de São Paulo, v.13, n.1, p. 46-55, 2003.

BRASIL. Ministério da Saúde. Conselho Nacional de Saúde. Resolução 196 de 10 de outubro de 1996. Diretrizes e normas regulamentadoras de pesquisa envolvendo seres humanos.Brasília.1996.

BRASIL. Ministério da Saúde. Secretaria de Políticas de Saúde. Departamento de Ações Programáticas Estratégicas. Plano de reorganização da atenção à hipertensão arterial e ao diabetes mellitus. Brasília (DF), 2001.

Disponível

em: http://dtr2001.saude.gov.br/sps/areastecnicas/cnhd/publi cacoes/doc/miolo2002.pdf. Acesso em 30 jul 2003.

BRASIL. Ministério da Saúde. III Consenso Brasileiro De Hipertensão Arterial. Hipertensão Arterial: disgnóstico e Classificação. Brasília (DF), 2001. Capítulo I. Disponível em:

http://dtr2001.saude.gov.br/bvs/publicacoes/III consenso bras hip arterial.pdf. Acesso em: 15 jan.2004.
CAMPOS JR., R.; COLOMBARI, E.; CRAVO, S.; LOPES, O. U. Hipertensão arterial: o eu tem a dizer o sistema nervoso. Rev. Bras Hipertens. Vol 8, n1, p. 4154, 2001. Disponível em http://departamentos.cardiol.br/dha/publicacoes/81/006.pdf. Acesso em: 10 dez. 2001.

IRIGOYEN, M. C.; LACCHINI, S.; De ANGELIS, K.; CICHELINI, L. C. Fisiopatologia da hipertensão: o que avançamos? Rev. Soc. Cardiol. Estado de São Paulo. V.13, n.1, p. 20-45, 2003.

LESSA, I. O adulto brasileiro $e$ as doenças da modernidade: epidemiologia das doenças crônicas nãotransmissíveis. São Paulo-Rio de Janeiro: Hucitec Abrasco,1998.

LOPES H, F. Hipertensão arterial e síndrome metabólica: além da associação. Rev. Soc. Bras. Card. v.13, n.1, p. 64-77, 2003.

MINAYO, M. C. S. O desafio do conhecimento: Pesquisa qualitativa em saúde. $7^{\mathrm{a} e d}$. São Paulo - Rio de Janeiro: Hucitec-Abrasco, 2000.

MINAYO, M. C. S. (org). Pesquisa Social: teoria, método e criatividade. 19a ed. Petrópolis: Vozes, 2001.

NETTINA, S.M. Prática de enfermagem. $7^{\mathrm{a}} \mathrm{ed}$. Rio de Janeiro: Guanabara Koogan, 2003.

NIH - NATIONAL INSTITUTES OF HEALTH..Seventh Report Of The Joint National Committee On Prevention, Detection, Evaluation, And Treatment Of High Blood Pressure. NIH Publications. n. 03-5231, 2003. Disponível em:

http://www.nhlbi.nih.gov/guidelines/hypertension/phycard pdf. Acesso em: 10 dez.2003.

OPARIL S. Hipertensão arterial. In: BENNETT, J. C.; PLUM, F. et al. Cecil - Tratado de medicina interna $20^{\mathrm{a} e d}$. Rio de Janeiro: Guanabara Koogan, 1997. v.1, p. 285-301.

ROUQUAYROL, M.Z; ALMEIDA FILHO, N. Epidemiologia, história natural e prevenção de doenças. In: ROUQUAYROL, M.Z; ALMEIDA FILHO, N.

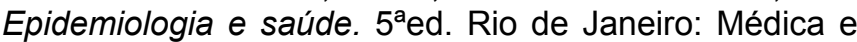
Científica, 1999. Capítulo. I, p. 15-31.

RUDIO, V. F. Introdução ao projeto de pesquisa científica $9^{\mathrm{a} e d .}$ Petrópolis: Vozes, 2001.

SILVA, J.L.L. Estilo de vida docente $x$ fatores de risco para hipertensão arterial sistêmica. 2004.157f. Trabalho de conclusão de curso (Graduação em Enfermagem e Licenciatura)- Escola de Enfermagem Aurora de Afonso Costa, Universidade Federal Fluminense. Niterói, 2004.

TOBAR, F; YALOUR M.R.Como fazer teses em saúde pública - conselhos e idéias para formular projetos e redigir teses e informes de pesquisa. Rio de Janeiro: Fiocruz, 2002.

Texto recebido em 10/09/2004

Publicação aprovada em 10/12/2004. 